\title{
Waking performance decrements following minimal sleep disruption: The effects of habituation during sleep
}

\author{
T. E. LeVERE, GERALD W. MORLOCK and F. D. HART \\ Department of Psychology and Department of Mechanical and Space Engineering \\ North Carolina State University, Raleigh, North Carolina 27607
}

\begin{abstract}
The present research was concerned with whether the occurrence of habituation to auditory stimuli during sleep might attenuate the disruptive effects these stimuli can have on waking performance. Human subjects were exposed on different nights to either 0,6 , or 24 presentations of a 15 -sec burst of filtered auditory noise, and their morning waking performance was measured by a reaction-time task 24 stimuli were presented, the to minimal sleep disturbances. The results indicated that on nights when the nights when onse significantly less as compared to However, the decreased average arousal when 6 , thus demonstrating the occurrence of habituation. morning performance. On the contrary, when 24 stimuli occurred was not associated with superior when only 6 stimuli . was significantly greater. The data thus the average arousal associated with the stimulus presentations performance is a fisruption and waking also, and perhaps more importantly, the number aroduced by the individual stimulus occurrences but
\end{abstract}

Recent evidence has suggested that minimal sleep disturbances can have effects on waking behavior and that it is possible to detect these effects with a simple reaction-time memory task soon after an individual awakens (LeVere, Bartus, \& Hart, 1972). Since waking effects following anything less than total sleep deprivation are somewhat of an oddity, it is of some importance to delineate the sleep parameters which may be relevant to the waking performance deficits noted by LeVere et al. (1972). The importance of this delineation is not only related to understanding why a particular waking behavior may be disrupted by sleep disturbances but also to a more general appreciation of the functional aspects of sleep itself. In this regard, two elementary questions may be advanced. The first concerns the individual's reaction to the stimuli which may disturb his sleep. For example, is it the magnitude of an individual's response to a particular stimulus presentation, or alternatively, is it the total amount of sleep disruption, independent of particular responses, which is the important parameter precipitating the waking effects? A second but related question concerns whether waking performance may correlate differentially with disturbances occurring during different electrophysiologically definable types of sleep? In more testable terms, these questions ask whether more arousing but less frequent stimuli might cause a greater decrement in waking behavior than less arousing but more frequent stimuli and might these effects interact

This research was supported by the National Aeronautics and Space Administration Research Grant No. NGL 34-002-095. T. E. LeVere and G. W. Morlock are members of the Department of Psychology and F. D. Hart is a member of the Department of Mechanical and Aerospace Engineering. The authors wish to thank C. LeVere and J. Black for their assistance in preparing this report. Reprint requests should be addressed to T. E. LeVere, Department of Psychology, North Carolina State University, Raleigh, North Carolina 27607. with the type of sleep being disturbed?

While there are perhaps a number of ways to approach these questions, an obvious method would be simply to vary the number of stimuli presented during the night. Since sleep does not necessarily preclude the occurrence of habituation (Firth, 1973), a greater number of stimuli should produce a smaller average subjective response than a more restricted number of stimulus occurrences. Thus, it should be possible to hold the physical parameters of the individual stimulus presentations constant and, by varying the number of stimuli presented during sleep, investigate the relation between response magnitude and waking performance. Moreover, if the stimulus presentations are tagged on the basis of sleep type, it should be also possible to correlate the disturbance of particular types of sleep with waking performance by simply testing for interaction effects.

\section{METHOD}

\section{Subjects}

The subjects were six paid male volunteers from the student population at North Carolina State University, ranging in age from 19 to 27 years (mean $=21.8$ years). None were under the care of a physician or taking any medication during their participation in the experiment. With the exception of sleeping in the laboratory, all subjects were instructed to maintain their normal daily routines excluding, of course, the use of alcohol or other narcotics.

\section{Apparatus}

The apparatus has been described previously (LeVere, Bartus, Morlock, \& Hart, 1973; LeVere, Morlock, Thomas, \& Hart, 1974 ) and need not be discussed in detail. Suffice it to say that the subjects slept in a laboratory mock-up bedroom constructed according to standard building practices and located within a larger laboratory area in a quiet [ambient noise $<40 \mathrm{~dB}(\mathrm{~A})$ ] portion of the building.

The auditory stimulus used to disturb sleep was a 15 -sec burst 
of random noise filtered to reject all frequencies save a $1 / 3$-octave band centered on the frequency of $125 \mathrm{~Hz}$. This sound was produced by a General Radio random noise generator (Model 1382), a Bruel and Kjoer audio frequency spectrometer, and a standard commercial audio amplifier coupled to an acoustic suspension speaker. The intensity of the stimuli was adjusted to a level of $80 \mathrm{~dB}$ measured with an A-weighted network from the general location of the subject's head during sleep.

The subject's scalp-recorded electroencephalographic (EEG) activity was utilized as both a dependent measure of the individual's response to the auditory stimuli and to insure that the individual stimulus occurrences were evenly distributed over different types of sleep occurring during the night. The EEG was obtained monopolarly from a Grass $9-\mathrm{mm}$ silver cup electrode attached to the P3 location (International 10-20 System) and referenced to the unilateral ear lobe. Rapid eye movements (REM) were detected with a similar electrode attached to the outer canthus of the ipsilateral eye and referenced to the same ear lobe. A fourth electrode was attached to the subject's neck for grounding. This electrode array obviously varies from the more extensive configuration suggested by Rechtschaffen and Kales (1968). However, this latter configuration is principally necessary to facilitate the categorization of sleep into standardized sleep stages on the basis of EEG, EOG, and EMG activity. Since the present research was primarily concerned with only distinguishing sleep characterized by nonREM fast-wave EEG activity and sleep characterized by slow-wave EEG activity and not by any subtle subdivision within these categories, it was decided that a more limited electrode array would be adequate.

The EEG activity was amplified by a Grass Model 79 polygraph set to bandpass filter below $1 \mathrm{~Hz}$ and above $1 \mathrm{kHz}$. From the polygraph, the signal was again bandpass filtered below $1 \mathrm{~Hz}$ and above $35 \mathrm{~Hz}$ and fed to a Digital Equipment Corporation PDP 12A computer system for on-line data analysis.

The PDP 12A computer system was programmed for the zero-crossings frequency analysis described by LeVere et al. $(1972,1973,1974)$. In general, this procedure digitizes the analogue signal at the rate of 200 points per second to prevent frequency aliasing and computes the occurrence of individual frequencies on the basis of the time elapsing between successive crossings of zero potential. The program further reduces this frequency data by accumulating wave occurrences within the standard EEG frequency categories of delta $(0-3 \mathrm{~Hz})$, theta $(4-7 \mathrm{~Hz})$, alpha $(8-12 \mathrm{~Hz})$, and beta $(13-35 \mathrm{~Hz})$, as these occur within successive 40 -sec analysis epochs separated by 20 -sec intervals to allow computer $\mathrm{I} / \mathrm{O}$. The number of waves occurring within the four categories was used to determine the electroencephalographic character of the subject's sleep for stimulus presentations. The major dependent measure to assess the subject's response to the auditory stimulation was the total number of half-wave occurrences during a given analysis epoch, i.e., the overall level of cortical desynchronization. This particular dependent measure was chosen for two reasons. Firstly, there is a relatively clear direct relationship between increases in cortical desynchronization (increases in the frequency pattern of the electroencephalogram) and arousal (Adrian \& Matthews, 1934; Moruzzi, 1965; Rheinberger \& Jasper, 1937; Sokolov, 1960). Secondly, the total number of half-wave occurrences within a standard $40-\mathrm{sec}$ analysis epoch does not seriously violate the assumptions of standard parametric statistical procedures.

The behavioral task used in the present procedures is illustrated in Figure 1. This task employs three vertically mounted 5/8-in. stimulus lights separated from one another by 1 in., center to center. These stimulus lights were at approximately eye level when the subject was seated at the apparatus. Four inches below these stimulus lights were three horizontally mounted 5/8-in. response buttons spaced 1-3/4 in.

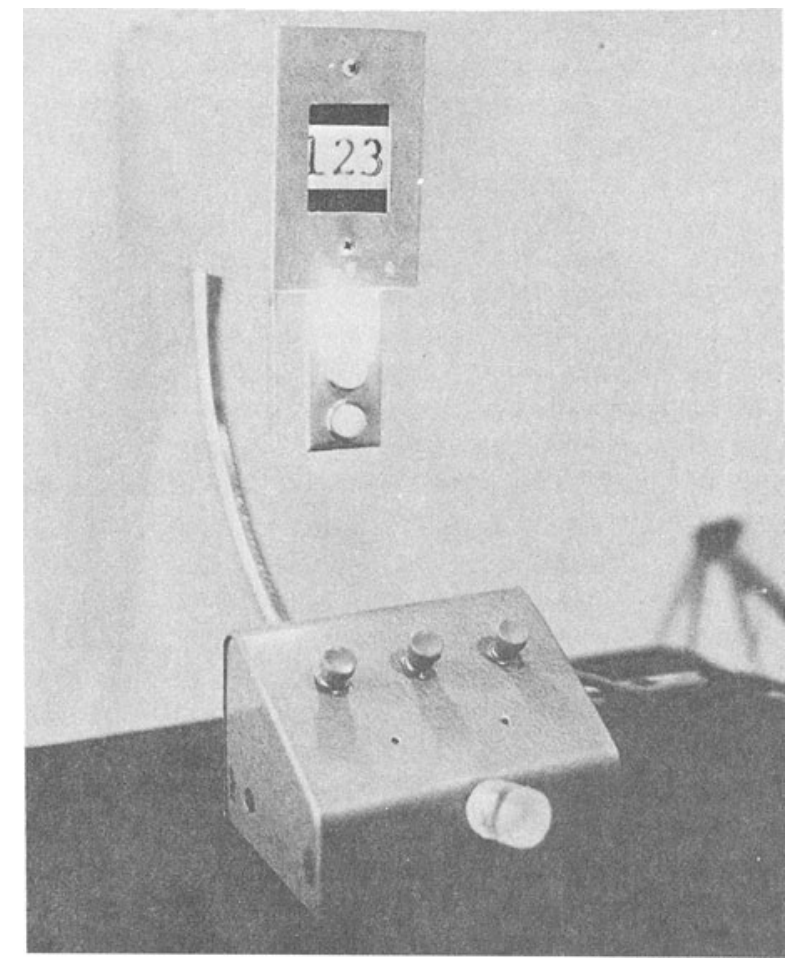

Figure 1. Photograph of performance task. See text for specifications and operational details.

apart, center to center. Some 2 in. below the center response button was a $1 \times 1 / 2$ in.-wide registration lever used by the subject to indicate that a single button had been pressed and to prevent double responding. The correspondence between the stimulus lights and the response buttons was determined by one of the six possible combinations (codes) of the numbers 1,2 , and 3. Individual codes were displayed from an Industrial Electronic Engineer readout display mounted above the stimulus lights. The manner in which the particular codes defined the relationship between individual stimulus lights and response buttons is easily visualized if the individual code numbers from left to right are superimposed upon the individual stimulus lights from top to bottom. The particular code number associated with a particular stimulus light then indicates the left-to-right position of the "correct" response button when a particular stimulus light is illuminated. For example, the code " 123 " indicates that the left-most response button is "correct" if the top stimulus light is illuminated, the middle response button if the middle stimulus light is illuminated, and the right-most button if the bottom stimulus light is illuminated. The other relationships between the individual stimulus lights and response buttons are similarly defined by the remaining five codes.

The operational characteristics of this task required that the subject visually monitor the three vertical stimulus lights and, when one was illuminated, press the response button indicated by the code and signal this choice by pressing the registration lever. If the subject pressed the appropriate or "correct" response button, the stimulus light was extinguished and the intertrial interval started. If the subject's response was "incorrect," the stimulus light remained on until the subject corrected his error by pressing the appropriate response button and registering this choice. While any given code was in effect for 
between 5 and 20 successive stimulus light presentations or trials, it must be emphasized that the code was displayed to the subject on only the first trial of the series of trials that it was in effect. Thus. in order for the subject to respond optimally, he must. after the first trial of a particular series, remember which code is in effect. It should be noted further that, since the subject was allowed as much time as needed to respond to the correct response button. the task was essentially subject-paced. The basic dependent measure was, of course, mean response latency as measured from stimulus-light onset to correct response and was computed over a standard 10 -min performance session.

\section{Procedure}

The experiment consisted of two distinct phases: a phase during which the subject learned to perform the behavioral task and a phase where the effect of nocturnal auditory stimuli on the performance of the task was evaluated. The first phase required that the subject come to the laboratory for 5 successive days (Monday-Friday) of the week just prior to the three nights which the subject was to sleep in the laboratory. On each of these days, the subject practiced the task: for two standard 10 -min sessions with a 5 - to 10 -min break betwe in ressions. At the end of each session, the subject was provided infor $m_{4}+$ ion concerning his performance and mildly encouraged to better his score on the next practice session (i.e., shorten his response latency and decrease the number of incorrect responses). The purpose of these practice sessions was to eliminate any learning component from the subject's performance of the behavioral task during the sleep phase of the experimental procedure.

During the sleep phase of the experimental procedure, each subject slept for three successive nights (Tuesday-Thursday) in the laboratory mock-up bedroom. These three nights were distributed between a single no-stimulus control night, a stimulus night when the subject was exposed to 6 auditory stimuli, and a stimulus night when the subject was exposed to 24 auditory stimuli. The order of occurrence of the single no-stimulus control night and two stimulus nights was balanced across the six subjects to control for the "first night" effect and other order effects. Each night which the subject spent in the laboratory, independent of whether it was a no-stimulus control night or a stimulus night, was divided in to $36040-\mathrm{sec}$ analy sis epochs, each of which was separated by a 20 -sec interepoch interval. The subject's sleep was not, however, restricted to this 6-h data-acquisition period. On the nights when stimuli were presented, each presentation was $15 \mathrm{sec}$ in duration, had an instantaneous onset and offset, commenced at the initiation of an analysis epoch, and was in accord with a predetermined schedule which evenly distributed the presentations over the 6-h data-acquisition period. There were, however. three restrictions on stimulus presentations: (1) half of the stimulus presentations were to occur when the subject's sleeping EEG pattern exhibited relatively fast-wave activity without REM and half were to occur when the subject's EEG pattern exhibited relatively slow'wave activity. (2) all stimulus presentations were to be separated by at least $5 \mathrm{~min}$, and (3) no stimulus presentations were to occur if REM occupied $10 \%$ is more of either of the two immediately preceding 40-sec analy sis epochs. The latter restriction reflects the fact that dreaming of:en accompanies REM activity and that extrinsic stimuli may be incorporated into the dream content (Freemon, 1972; Rechtschaffen, Hauri, \& Zeitlin, 1966) which makes subjective responsiveness largely a matter of definition and obviously difficult to evaluate with the dependent measure of cortical desynchronization.

The determination of the two sleep categories, nonREM fast-wave sleep and slow-wave sleep, as well as wakefulness, was made on-line by the PDP 12A computer. Slow-wave sleep was defined by the occurrence of $20^{\prime} c$ or more delta EEG activity within an analysis epoch. NonREM fast-wave sleep was scored when the EEG record indicated less than $20 \%$ delta activity and additionally less than $20, c$ alpha and beta activity. Wakefulness was assumed when the frequency pattern of the recorded EEG activity fell within neither of these classifications. The computer classification of wakefulness, nonREM fast-wave sleep, and slow-wave sleep according to these criteria has been previously validated by LeVere et al. (1974).

Before retiring for the night, each subject was required to perform the behavioral task for the standard $10-\mathrm{min}$ performance session. These performance sessions were identical to the practice sessions of the previous week's training. After the subject completed the performance session, he was settled in bed and the electroencephalographic and eye-movement recording started. From this point and throughout the remainder of the night, the experiment was under computer control. The 6-h data-acquisition period was begun when the computer sensed the first 5 uninterrupted minutes of sleep as previously defined. The first stimulus was scheduled to occur $5 \mathrm{~min}$ after this, provided the previously described restrictions were met. Regarding these presentations, three points should be made explicit. Firstly, the auditory stimuli were presented at the initiation of a $40-\mathrm{sec}$ analysis epoch so that the presentation of a stimulus was necessarily determined with respect to the two epochs just preceding the epoch during which the stimulus occurred. Secondly, since slow-wave delta sleep is more predominant during the early portion of a night's sleep, this type of sleep was given priority over fast-wave sleep. And thirdly, if three successive analysis epochs were expended trying to meet the presentation restrictions, the stimulus was presented by default during the fourth analysis epoch in order that the correct number of stimuli would occur during each night. If REM was not present during the two epochs prior to the time the stimulus was presented, the computer stored the number of half-wave occurrences within the delta, theta, alpha, and beta frequency categories as well as the total number of half-wave occurrences for: (1) the 40-sec analysis epoch preceding the stimulus presentation, (2) the 40-sec analysis epoch during which the stimulus occurred, and (3) the 40-sec analysis epochs succeeding the presentation of the stimulus. The no-stimulus control nights were programmed in exactly the same manner except. of course, that no auditory stimuli were presented during the night.

Upon awaking in the morning, the subject was again required to perform the behavioral task. This performance session was identical to that occurring prior to sleep the night before. It must be emphasized, though, that that morning performance session was not started until the subject himself determined that he was fully awake, alert, and able to effectively work. While this had the potential of introducing some variability into the experimental procedure, it was decided that this would be more desirable than forcing the subjects to perform when they were not ready to do so. In fact, however, the starting time was quite consistent across the subjects, since all elected to begin the task within 10 min after they were awakened.

\section{RESULTS}

\section{Arousal During Sleep}

Figure 2 presents the average arousal response during sleep produced by the $1 / 3$-octave band of noise centered on the frequency of $125 \mathrm{~Hz}$. The figure organizes the data according to whether the stimuli were presented during sleep characterized by nonREM fast-wave EEG activity (left side) or sleep characterized by slow-wave EEG activity (right side) and, additionally, whether 
there were 24,6 , or no stimulus presentations during the night. The solid horizontal line at the top of the figure labeled "wakefulness" indicates the level of cortical desynchronization that was recorded during the analysis epoch just preceding the five successive analysis epochs which defined the onset of sleep. The horizontal dashed lines above and below this solid line represent the .05 confidence interval associated with this rather conservative estimate of wakefulness.

In general, the data presented in this figure clearly suggest that a 15 -sec burst of filtered auditory noise at a level of $80 \mathrm{~dB}(\mathrm{~A})$ is an effective stimulus for arousal during sleep in terms of increases in the frequency content of the individual's EEG activity (cortical desynchronization). Moreover, it makes little difference whether this arousal is calculated relative to the analysis epoch just preceding the occurrence of the auditory stimulation or relative to the no-stimulus control night. There are, however, two things concerning this arousal which should be specifically noted. Firstly, the auditory stimuli appeared quite a bit more effective during sleep characterized by slow-wave EEG activity than during sleep characterized by nonREM fast-wave EEG activity. Secondly, the mean arousal, in terms of cortical desynchronization, was below that associated with wakefulness. Thus, while the stimuli were effective in terms of changing the ongoing electroencephalographic activity during sleep, they were not equally effective during all types of sleep and were quite ineffectual in consistently producing behavioral awakening.

While these results are of some interest, if only to replicate other research, what is more important is the relative arousal which occurred when 6 stimuli were presented during the night and when 24 stimuli were presented during the night. As can be seen from Figure 2, the average response to the auditory stimuli was much greater when only 6 stimuli occurred during the night as compared to when 24 stimuli occurred during the night. Moreover, the greater responsiveness to the auditory stimuli during six-stimulus nights did not appear to be selective to either sleep characterized by nonREM fast-wave EEG activity or sleep characterized by slow-wave EEG activity. Thus, it would seem that the present experimental procedure was compatible with the phenomenon of habituation and would allow an evaluation of how level of arousal vs. number of arousals may influence waking performance.

Statistically, these conclusions are supported by an overall $\mathrm{F}$ ratio which was significant for the third-order interaction $(F=3.97, \mathrm{df}=8 / 40, \mathrm{p}<.01)$ and for the second-order interactions associated with the data obtained during nonREM fast-wave sleep $(F=3.52$, $\mathrm{df}=8 / 40, \mathrm{p}<.01)$ and the data obtained during slow-wave sleep $(F=5.19, \quad d f=8 / 40, \quad p<.01)$. Individual comparisons may thus be determined on the

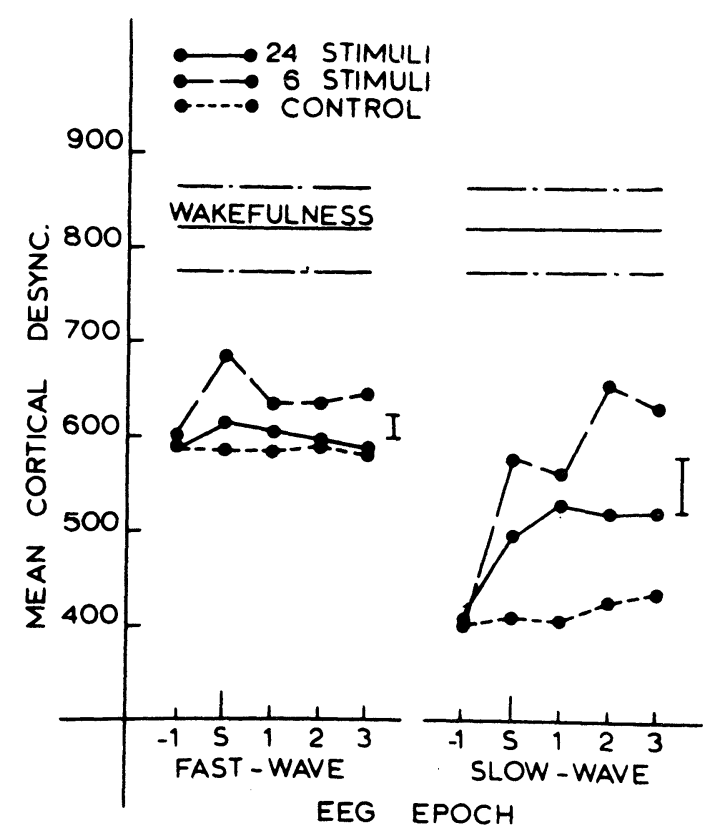

Figure 2. Mean cortical desynchronization (arousal) recorded $1 \mathrm{~min}$ prior to the occurrence of a stimulus, during the presentation of the stimulus, and for 3 succeeding minutes following the stimulus occurrence. The figure presents separate curves for the nights when no stimuli were presented, for nights when 6 stimuli were presented, and for nights when 24 stimuli were presented and groups these curves according to whether the stimuli occurred during nonREM fast-wave sleep or slow-wave sleep. The small vertical lines associated with each group of curves represent the .05 critical difference value for that group of curves. The solid horizontal line labeled wakefulness is the mean cortical desynchronization recorded just $1 \mathrm{~min}$ prior to sleep onset. The horizontal dashed lines associated with this solid line are the .05 confidence limits of this data.

basis of the critical difference value which is depicted by the small vertical line associated with each group of curves.

\section{Waking Performance}

The intended effect of the practice sessions to eliminate learning from the performance of the behavioral task during the sleep portion of the experiment was not altogether successful. Figure 3 presents the performance data (response latency) during the five practice sessions as well as during the evening performance sessions during the sleep portion of the experiment. The data are presented by days and do not reflect the effects of the experimental conditions during the sleep portion of the experiment. As can be seen, there was a consistent decrease in response latency during the practice sessions and during the evening performance sessions of the sleep phase of the experiment. While this decrease in response latency was quite a bit greater during the practice sessions, as would be expected, it had still not stabilized by the time the 


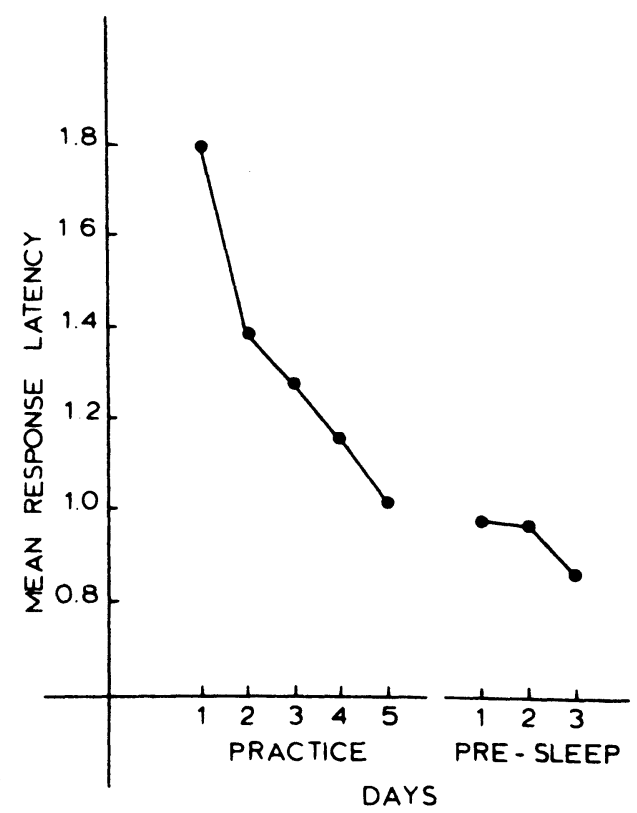

Figure 3. Mean response latency in seconds as computed over the 5 practice days and during the evening performance sessions in the sleep portion of the experimental procedure.

subject participated in the sleep portion of the experiment. Statistically, these trends are significant as indicated by an analysis of variance computed over all the performance sessions $(F=66.71, d f=7 / 35, p<.01)$ and an analysis of variance restricted to the evening performance sessions during the sleep portion of the experiment $(F=9.82, \mathrm{df}=2 / 10, \mathrm{p}<.01)$. This result then makes the evaluation of the effects of sleep disruption difficult, if not inappropriate, in terms of absolute morning performances following the different nocturnal stimulus conditions. However, it is possible to stabilize the data and control for this learning component by analyzing the effects of the experimental treatments in terms of the change in morning performance relative to the prior evening's performance.

Figure 4 presents this analysis utilizing the difference between the response latency recorded during the evening performance session and the response latency recorded during the morning performance session for the three treatment conditions. Since the response latency during morning performances was always longer than during evening performances, the evening scores were subtracted from the morning scores in order that the differences might be positive numbers. Inspection of the figure indicates that there was little difference between undisturbed sleep and the occurrence of six 15-sec 80- $\mathrm{dB}(\mathrm{A})$ stimuli during the night. However, when the number of stimuli was increased to 24 , there appeared a four-fold increase in response latency during the morning performance session. Statistically, this difference in morning performance is confirmed by an analysis of covariance using the evening performance score as the covariant $(F=4.294, d f=2 / 14, p<.05)$.

Because of the way response latency was computed, i.e., from stimulus-light onset to the occurrence of a correct response, it is obvious that the noted increase in response latency could be due to either a genuine increase in response latency or to the occurrence of errors prior to a correct response. To distinguish between these two possibilities, an analysis of variance was computed on the number of first-choice-correct responses and on the number of errors committed during the morning performance sessions following the three treatment conditions. Analyses were performed both in terms of the absolute values of these measures and relative to the previous night's performance. In all cases, the F-ratios were nonsignificant. This suggests that the behavioral degradation following sleep disturbed by 24 stimulus presentations was not a function of some change in the individual's accuracy but rather reflected a general slowing of whatever processes were required for optimal performance.

To relate waking performance more specifically to sleep disruption, an attempt was made to estimate the overall quality of sleep by determining the amount of

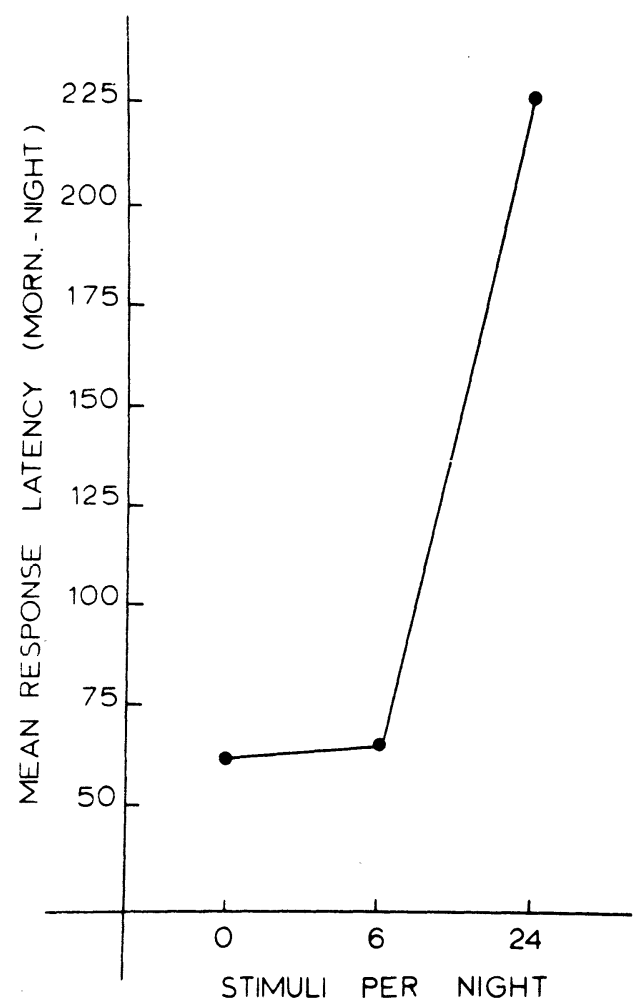

Figure 4. Mean response latency during morning performance sessions following nights undisturbed by auditory stimuli, following nights disturbed by 6 stimulus presentations, and following nights disturbed by 24 stimulus presentations. 
sleep disruption occurring during a given night. This analysis involved computing the subject's response to an auditory stimulus by taking the level of cortical desynchronization (arousal or number of half waves) recorded just prior to an auditory stimulus and subtracting this value from the level of cortical desynchronization recorded during: (1) the analysis epoch when the stimulus occurred, and (2) the next three succeeding analysis epochs. These difference scores were then summed to estimate, within the limits of the present data, the total effect of a given auditory stimulus. Following this, the totals for each stimulus occurrence were then summated over a given night to estimate the total sleep disruption associated with the occurrence of either 6 or 24 stimulus presentations. Using this data, there was an average total sleep disruption (total cortical desynchronization) of 33.2 half waves when 6 stimuli were presented, while during nights when 24 stimuli were presented, this average jumped to 601.1 half waves. The difference between these values is significant at the .05 level of confidence $(\mathrm{F}=7.54, \mathrm{df}=1 / 5)$. Thus, sleep disruption, in terms of total change in cortical desynchronization, was significantly less when 6 stimuli were presented as compared to when 24 stimuli were presented even though the stimulus presentations in this latter instance precipitated a significant amount of habituation.

While the above analysis may simply reaffirm the obvious-greater sleep disruption produces greater waking effects-what is not so obvious, and perhaps more interesting, is that the relationship between total sleep disruption and waking performance is not the same for sleep characterized by nonREM fast-wave EEG activity and sleep characterized by slow-wave EEG activity. This is shown by the interaction depicted in Figure 5, which illustrates the total sleep disruption produced by the auditory stimuli during nonREM fast-wave sleep and during slow-wave sleep when 6 or 24 stimuli occurred during the night. The interaction is, of course, significant $(F=19.27, \mathrm{df}=1 / 5, \mathrm{p}<.01)$, and what is immediately apparent is that there is little difference in total sleep disruption during fast-wave sleep when 6 stimuli were presented and when 24 stimuli were presented during the night. However, during sleep characterized by slow-wave EEG activity, there was significantly greater total sleep disruption during the nights when 24 stimuli occurred as compared to when only 6 stimuli occurred. And, it is this differential disruption during slow-wave sleep which is most congruent with the observed decrement in waking performance. Thus, using total cortical desynchronization as a criterion for sleep disruption, slow-wave sleep would appear more critical than nonREM fast-wave sleep in terms of optimal waking performance.

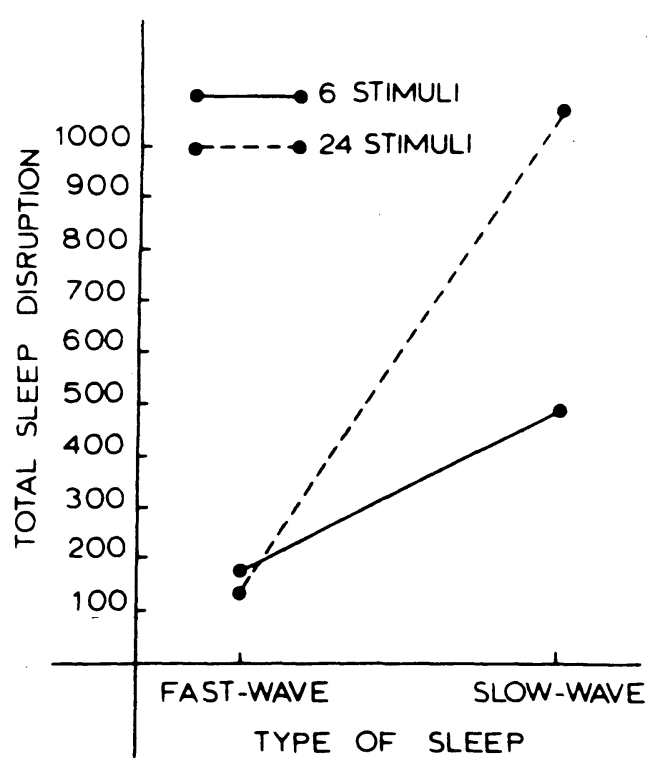

Figure 5. Plot of the differential amounts of total sleep disruption (summed arousal overa 11 stimulus presentations) occurring during nonREM fast-wave sleep and during slow-wave sleep when 6 and 24 stimuli were presented during the night. The interaction is significant with the total sleep disruption occurring not reliably different for 6 or 24 stimulus presentations during nonREM fast-wave sleep but significantly greater for 24 stimulus presentations during slow-wave sleep.

\section{DISCUSSION}

Considering first the arousal from sleep produced by the auditory stimuli, the present results generally replicate previous researches indicating that similar auditory stimuli produce greater cortical desynchronization during sleep characterized by slow-wave EEG activity than during sleep characterized by nonREM fast-wave EEG activity (LeVere et al., 1973, 1974). This aspect of the present data is thus a reaffirmation of the now-rather-general consensus that it is no longer tenable to consider slow-wave sleep a "deeper" sleep or that the subject is less responsive during this type of sleep (Williams, Holloway, \& Griffiths, 1973).

Turning more specifically to the question of habituation, the present data suggest that the subject's response to the individual stimulus presentations was significantly greater when only 6 stimuli occurred during the night as compared to when 24 stimuli occurred during the night. This occurrence of habituation is somewhat interesting in the present situation, since the conditions governing the stimulus presentations were not what would normally be considered optimal for habituation. That is, not only were the intervals between stimulus presentations quite long (over $3 \mathrm{~min}$ ) but they were also quite variable. In fact, the occurrence of an 
auditory stimulus was not only dependent upon a variable-interval presentation schedule but also upon the particular electroencephalographic pattern of the sleeping individual. Furthermore, it does not appear that habituation is restricted to either sleep characterized by nonREM fast-wave EEG activity or to sleep characterized by slow-wave EEG activity but rather seems to operate with equal facility during either type of sleep. Thus, habituation during sleep may not be as elusive a phenomenon as has previously been suggested (Firth, 1973; Johnson \& Lubin, 1967) but may, like so many other parameters of sleep, depend upon how its occurrence is signaled.

Regarding waking behavior, however, the greater arousal response occurring when only six stimuli were presented during the night was not associated with a greater deficit in morning performance. Rather, the subject's morning performance appeared more related to the total number of stimulus presentations which occurred during the night. However, it must be pointed out that when the subjects were questioned in the morning, they were unable to recall the number of stimuli which had been presented during the night, and were simply aware that some auditory stimuli had occurred. This result leads to two conclusions concerning the observed morning performance decrement. Firstly, since the subjects were unable to determine the number of auditory stimuli which were presented during the night, their subjective evaluation of the quality of their sleep most likely did not precipitate the differential morning performances following different amounts of nocturnal stimulation. The performance deficit is thus more probably related to the disruption of sleep per se and not any sort of subjective justification of less effort because there was more noise on one stimulus night than on another stimulus night. This point is even more salient when it is remembered that there was virtually no difference between performances following no-stimulus control nights and performances following stimulus nights when six stimuli were presented. That is, the subjects knew that their sleep was disturbed by auditory stimulation but this did not differentially influence their morning performance.

The seond conclusion concerns the implication that, even though the subjects may show significant habituation, this habituation or decrement in nocturnal responsiveness does not necessarily mean that the stimuli were ineffectual. This is, however, not terribly surprising, since it is well known that even though an individual may not overtly respond to a particular stimulus, occurrences of this stimulus are nonetheless still registered by the individual. A case in point is the fact that a habituated response returns if the stimulus intensity is either slightly increased or decreased i.e., dishabituation can occur (Sokolov, 1960). That the greater number of stimuli in the present procedure were associated with a lower average EEG arousal response but yet were still capable of producing greater waking performance deficits may simply be another example of the fact that individuals still respond to stimuli which are, by usual criteria, supposedly ignored.

However, simply to explain the present results on the basis of some suggestion that an individual continues to respond to stimuli even after he apparently habituates is in some sense begging the question. Obviously, the individual must continue to respond to the extrinsic auditory stimulation or there would be no differential performance effects related to the number of stimuli presented during the night. The critical point is how stimuli which produce less arousal may precipitate greater performance deficits. On the basis of the present data and analysis, it would appear that the overall or total sleep disruption is a more important variable than the individual's response to the individual stimuli. This is not to say that the level of an individual's response to nocturnal stimulation is unimportant, but simply that there is probably a balance, or trade-off, between the arousal produced by the individual stimulus presentations and the number of stimuli which occur. This result is, of course, similar to a number of classic psychophysical relations with the Bunsen-Roscoe law and Ricco's law, concerning, respectively, temporal and spatial summation of visual stimuli, perhaps the most well known.

The differential total sleep disruption associated with sleep characterized by nonREM fast-wave sleep and slow-wave sleep and the correspondence of slow-wave sleep disruption to waking performance is somewhat more difficult to understand. A speculation, and the emphasis must be placed upon speculation, involves the recently formalized suggestion (Freemon, 1972; Hartmann, 1973) that sleep is a restorative process. Assuming this restorative function of sleep, Hartmann (1973) has further suggested that the absence of certain behaviors during sleep, or at least during certain types of sleep, may be taken as an indication of the restoration process and what is absent may tag the particular behavior patterns being restored. While there are a number of aspects of the present behavioral task, two which are certainly critical to optimal performance are: (1) a response to an extrinsic stimulus and (2) the mnemonic storage and retrieval of the code defining the stimulus-response relationships. Since simple reaction-time or vigilance tasks are notably insensitive to minimal sleep disruptions (Wilkinson, 1968), and since sleep does not preclude responding to extrinsic stimulation (see Oswald, 1962, as well as the present data), the response aspect of the present task may be somewhat unimportant. On the other hand, memory process appears to be sensitive to sleep disruption 
(Williams, Gieseking, \& Lubin, 1966), and if memory is at all operative during sleep, it is operative during fast-wave sleep but probably not operative during slow-wave sleep (Koukkou \& Lehmann, 1968). This, then, suggests that it may be the memory aspect of the present task which is critical and that it may be the interference of the restoration of memory processes during slow-wave sleep which precipitates the observed decrement in performance. However, before it is possible to espouse this speculation with any degree of confidence, considerably more evidence will be required. Nonethless, the present data do, if nothing else, reaffirm the commonly held belief that waking behavior should not be divorced from sleep.

\section{REFERENCES}

Adrian, E. D., \& Matthews, B. H. C. Berger rhythm: Potential changes from the occipital lobes in man. Brain, 1934, 57, 355-385.

Firth, H. Habituation during sleep. Psychophysiology, 1973, 10. 43-51.

Freem in, F R. Sleep research. Springfield: Thomas, 1972.

Hartmann, E. L. The functions of sleep. New Haven: Yale University Press, 1973.

Johnson, L. C., \& Lubin, A. The orienting reflex during waking and sleeping. Electroencephalography and Clinical Neurophysiology, $1967,22,11-21$.

Koukkou, M., \& Lehmann, D. EEG and memory storage in sleep experiments with humans. Electroencephalography and Clinical Neurophysiology, 1968, 25, 455-462.

LeVere, T. E., Bartus, R. T., \& Hart, F. D. Electroencephalographic and behavioral effects of nocturnally occurring jet aircraft sounds. Aerospace Medicine, 1972, 43, 384-389.
LeVere, T. E., Bartus, R. T., Morlock, G. W., \& Hart, F. D. Arousal from sleep: Responsiveness to different auditory frequencies equated for loudness. Physiology and Behavior, 1973, 10, 53-57.

LeVere, T. E., Morlock, G. W., Thomas, L. P., \& Hart, F. D. Arousal from sleep: The differential effect of frequencies equated for loudness. Physiology and Behavior, 1974, 12, 573-582.

Moruzzi, G. The functional significance of sleep with particular regard to the brain mechanisms underlying consciousness. In J. C. Eccles (Ed.) Brain and conscious experience. New York: Springer, 1965.

Oswald, I. Sleeping and waking. New York: Elsevier, 1962.

Rechtschaffen, A., Hauri, P., \& Zeitlin, M. Auditory wakening thresholds in REM and NREM sleep stages. Perceptual and Motor Skills, 1966, 22, 927-942.

Rechtschaffen, A., \& Kales, A. A manual of standardized terminology, techniques and scoring system for sleep stages for human subjects. Washington Public Health Service, U. S. Government Printing Office, 1968. P. 13.

Rheinberger, M., \& Jasper, H. H. Electrical activity of the cerebral cortex in the unanesthetized cat. American Journal of Physiology, 1937, 119, 186-196.

Sokolov, E. N. Neuronal models in orienting reflex. In M. A. B. Brazier (Ed.) The cerebral nervous system in behavior: Transactions of the 3rd conference. New York: Josiah Macy, Jr. Foundation, 1960.

Wilkinson, R. T. Sleep deprivation: Performance tests for partial and selective sleep deprivation. In $\mathrm{L}$. A. Abt and B. F. Riess (Eds.) Progress in clinical psychology. New York: Grune, 1968 .

Williams, H. L., Holloway, F. A., \& Griffiths, W. J. In P. H. Mussen and M. R. Rosenzweig (Eds.) Annual review of psychology. Palo Alto: Annual Reviews, Inc, 1973. Pp. 279-316.

williams, H. Lieseking, C. F., \& Lubin, A. Some effects of sleep loss on memory. Perceptual and Motor Skills, 1966, 23, $1287-1293$.

(Received for publication July 30,1974 ; revision received November $4,1974$. 